\title{
Dengue Fever-A Seasonal Malady
}

\author{
Ruksana Ahmed*, Fatima Amatullah, Jyothi Allamsette and Suneetha Padala \\ Department of Laboratory Medicine, Care Hospitals, Hyderabad, India \\ *Corresponding author
}

\section{A B S T R A C T}

\section{Keywords}

Period, Dengue, Lethal, Ecology, Environment,

Vector, Monsoon, Aedes

Article Info

Accepted:

07 October 2019

Available Online:

10 November 2019
Dengue fever, also known as breakbone fever, is a mosquito-borne infectious tropical disease caused by the dengue virus. Incidence of dengue is reported to be influenced by climatic factors. The objective and aim of this study is to map the monthly variation in the incidence of positive cases of dengue viral fever at a tertiary care hospital in Hyderabad. It was found that the lowest incidence of dengue fever was in the months -December 2018 to April 2019. 52.32\% of the total dengue cases for the period of study were seen in the months of July and August this corresponded to increased rainfall and moderate temperature. It was also seen that majority of the dengue cases i.e $70.43 \%$ were in patients below 30 years of age. It was further observed that Dengue viral fever was more prominent in males $(63.8 \%)$ as against female patients $(36.1 \%)$. The present study too found the efficacy of NS1 assay as a tool for early detection of dengue viral infection when used within 5 days of fever as also recommended by The National Guidelines for Clinical management of Dengue fever released by the Govt of India.

\section{Introduction}

Dengue, an acute viral infection with potentially lethal consequences, has been declared one of the most significant and prevalent arthropod-borne communicable diseases $^{1}$. It is the most extensively spread mosquito-borne disease, transmitted through the bite of infected mosquitoes of Aedes species. According to WHO report of 2009,
Dengue is endemic in more than 100 countries with most cases being reported from the Americas, South-East Asia and Western Pacific regions of WHO. In India, dengue is endemic in almost all states and is the leading cause of hospitalization ${ }^{2}$. The disease, which typically had an urban distribution a few decades earlier, is now reported from periurban as well as rural areas ${ }^{i}$. Studies from India have correlated increased dengue 
incidence with the monsoon and postmonsoon season ${ }^{2}$. There is scientific evidence that temperature and rainfall influence dengue incidence. However, complex interactions of ecology, environment, vector and virus serotypes are crucial factors driving dengue outbreaks $^{3}$.

The number of dengue cases has gradually increased in India. Dengue is driven by complex interactions among host, vector and virus that are influenced by climatic factors. The Incidence of dengue is reported to be high during rainy season in the Hyderabad district of Telangana state.

The main aim and objective of the present study is to

To determine the incidence of dengue fever during the monsoon months at a tertiary care hospital.

To study the age wise distribution of dengue fever patients

To serologically confirm acute dengue fever in clinical suspects

\section{Materials and Methods}

A total of 2520 serum samples were collected from patients presenting to the ER/OP with acute febrile illness and clinical suspicion of Dengue. The study was conducted for a duration of four months (June-2019 to September, 2019) during the monsoon period at a tertiary care hospital. The following criteria was used to enroll the study subjects. Inclusion criteria was patients with clinical suspicion of dengue(fever, myalgia, headache, retro bulbar pain) as per the WHO criteria and exclusion criteria was fever patients with other confirmed diagnosis like scrub typhus, leptospirosis, malaria, enteric fever or any other identified infection. All the above samples were screened to determine the presence of NS-1 antigen and IgM antibodies which are the serological markers of acute dengue fever using the following laboratory assays as per the NVBDCP guidelines:

Dengue Early ELISA (Panbio Dengue early ELISA, Standard Diagnostics, Inc., Gyeonggido, Republic of Korea)

Dengue IgM capture ELISA (Panbio Dengue IgM ELISA, Standard Diagnostics, Inc., Gyeonggi-do, Republic of Korea)

\section{Results and Discussion}

\section{The incidence and prevalence of dengue} viral fever across seasons

The present study was done at a tertiary care hospital at Hyderabad, during the period October 2018 to September 2019 with particular emphasis on the monsoon season i.e. the period from June 2019 to end of September 2019. In the study it was found that cases of acute dengue viral fever were reported during the whole of last one year but the number of positive cases of acute Dengue viral fever differed significantly during the post monsoon season, pre-monsoon season and the monsoon season. In the post monsoon season i.e. from the months October 2018 to January 2018 there were 151 cases of dengue viral fever, during the pre-monsoon period extending from February 2019 to May 2019 there were 71 positive for dengue viral fever; and during the monsoon season there were 900 positive cases for dengue viral fever.

From the monthly distribution chart below of the subjects, it is seen that the five month period from December 2018 to April 2019 the number of Dengue viral fever cases were the lowest. From May onwards the number of Dengue viral fever cases started increasing and peaked during the month of July 2019. The number of cases of Dengue viral fever for the months of July and August 2019 were 
$52.32 \%$ of the total dengue cases for the period -Oct 2018 to Sept 2019. The number of Dengue viral fever cases for the months of July 2019 to Sept 2019 amounts to $70.69 \%$.

From the above, it can be seen that the maximum number of patients were in the age group 20-29 (presumably Office goers) and the second highest incidence of dengue was in the age group 10-19 (teenagers) and after that the third highest incidence of dengue was in 0 9 age group (children). Thus a majority of the dengue cases i.e $70.43 \%$ were in patients below 30 years of age. It was further observed that Dengue viral fever was more prominent in males $(63.8 \%)$ as against female patients (36.1\%).

From the table 1 it is indicated that total number of patients attending the OPD /ER with acute febrile illness and clinical suspicion of dengue were 2520. A total of 900 samples were positive for dengue fever which were included in the study. Total samples received for Ns-1 were 1900 (75.39\%) and IgM 620 (24.60\%) for laboratory analysis from June2019 to Sept 2019. Among these samples the Dengue seropositivity and prevalence was $35.7 \%$. The non-structural protein (NS-1) which is an early serological marker of the infection showed a positivity of $30 \%$ while the IgM showed 53.2\%.

The highest seropositivity of NS-1 was observed in the month of July (40.0\%) while IgM also showed a highest positivity of (68.2\%) therefore indicating that it was in the month of July that dengue fever cases were high in patients attending our hospital.

The seasonal trend after reaching a peak gradually comes down in the month of September, however some intermittent sprouts in dengue fever are seen throughout the year. In a study done in Brazil in 2018, the correlation between rainfall and the occurrence of dengue cases showed that the number of cases increased in the first few months after the rainy season. In another study done in Taiwan in 2018, the result indicated that temperature, accumulated rainfall, and sunshine play an important role in the transmission cycles of dengue fever ${ }^{4}$. Similarly, in a detailed study done in 2019 in the city of Delhi, showed a clear dependency of dengue cases with climatic factors and further revealed that there is a lag phase between rains and appearance of cases ${ }^{4}$. In another study done in the city of Delhi a positive but weak correlation was found between the rainfall and incidence of dengue in Delhi ${ }^{5}$; whereas another study done by the same author found a strong positive correlation between the rainfall and incidence of dengue in Rajasthan ${ }^{6}$.

The present study too demonstrates a huge spike in case of Dengue viral fever in monsoon season from June 2019 to Sept 2019, when compared to pre-monsoon and post monsoon season. The incidence of Dengue viral fever was found to be lowest in months from December to March i.e the winter season while the highest number of Dengue viral fever cases was found in July (when the combination of temp and rainfall is relatively high in comparison to other monsoon months). However, the impact of high or low temperature on the incidence of Dengue viral fever needs to be studied further. In the present study it was also found that majority (70.43\%) of positive cases for Dengue viral fever were in the age group below 30 years. It was further observed that Dengue viral fever was more prominent in males $(63.8 \%)$ as against female patients $(36.1 \%)$. This is in line with the numbers reported by a 2018 study in a tertiary care hospital in Pune; wherein a total of 299 patients' records were assessed (Fig. 1 and 2; Table 2). 
Table.1 Age wise distribution of dengue fever patients

\begin{tabular}{|c|c|c|c|}
\hline SI No & Age group in years & Number of cases & Percentage \\
\hline $\mathbf{1 .}$ & $0-9$ & 188 & $20.88 \%$ \\
\hline $\mathbf{2 .}$ & $10-19$ & 210 & $23.33 \%$ \\
\hline $\mathbf{3 .}$ & $20-29$ & 236 & $26.22 \%$ \\
\hline $\mathbf{4 .}$ & $30-39$ & 157 & $17.44 \%$ \\
\hline $\mathbf{5 .}$ & $40-49$ & 56 & $6.22 \%$ \\
\hline $\mathbf{6 .}$ & More than 50 & 53 & $5.88 \%$ \\
\hline & & 900 & $100 \%$ \\
\hline
\end{tabular}

Table.2 The serological confirmation of dengue viral fever- data pertaining to the monsoon months of June 2019 to Sept 2019 is as tabulated below

\begin{tabular}{|c|c|c|c|c|c|c|c|c|}
\hline Month & $\begin{array}{c}\text { NS-1 } \\
\text { tested }\end{array}$ & $\begin{array}{c}\text { NS-1 } \\
\text { Positive }\end{array}$ & Percentage & & Month & $\begin{array}{c}\text { IgM } \\
\text { tested }\end{array}$ & $\begin{array}{c}\text { IgM } \\
\text { Positive }\end{array}$ & Percentage \\
\cline { 1 - 4 } June & 174 & 67 & $38.50 \%$ & & June & 42 & 25 & $59.52 \%$ \\
\hline July & 554 & 222 & $40.07 \%$ & & July & 126 & 86 & $68.25 \%$ \\
\hline August & 647 & 157 & $24.26 \%$ & & August & 259 & 133 & $51.35 \%$ \\
\hline Sept & 525 & 124 & $23.60 \%$ & & Sept & 193 & 86 & $44.55 \%$ \\
\hline Total & 1900 & 570 & $30 \%$ & & Total & 620 & 330 & $53.22 \%$ \\
\hline
\end{tabular}

Fig.1 The month wise distribution of dengue viral fever cases for the last one year is as follows

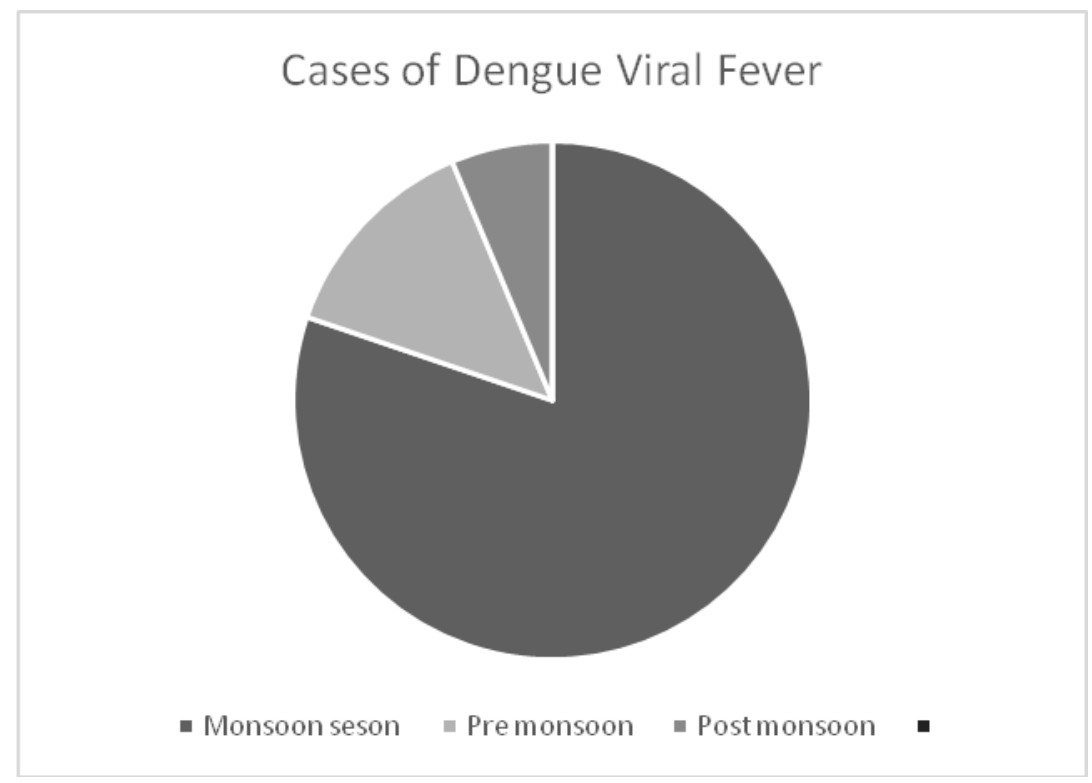


Fig.2

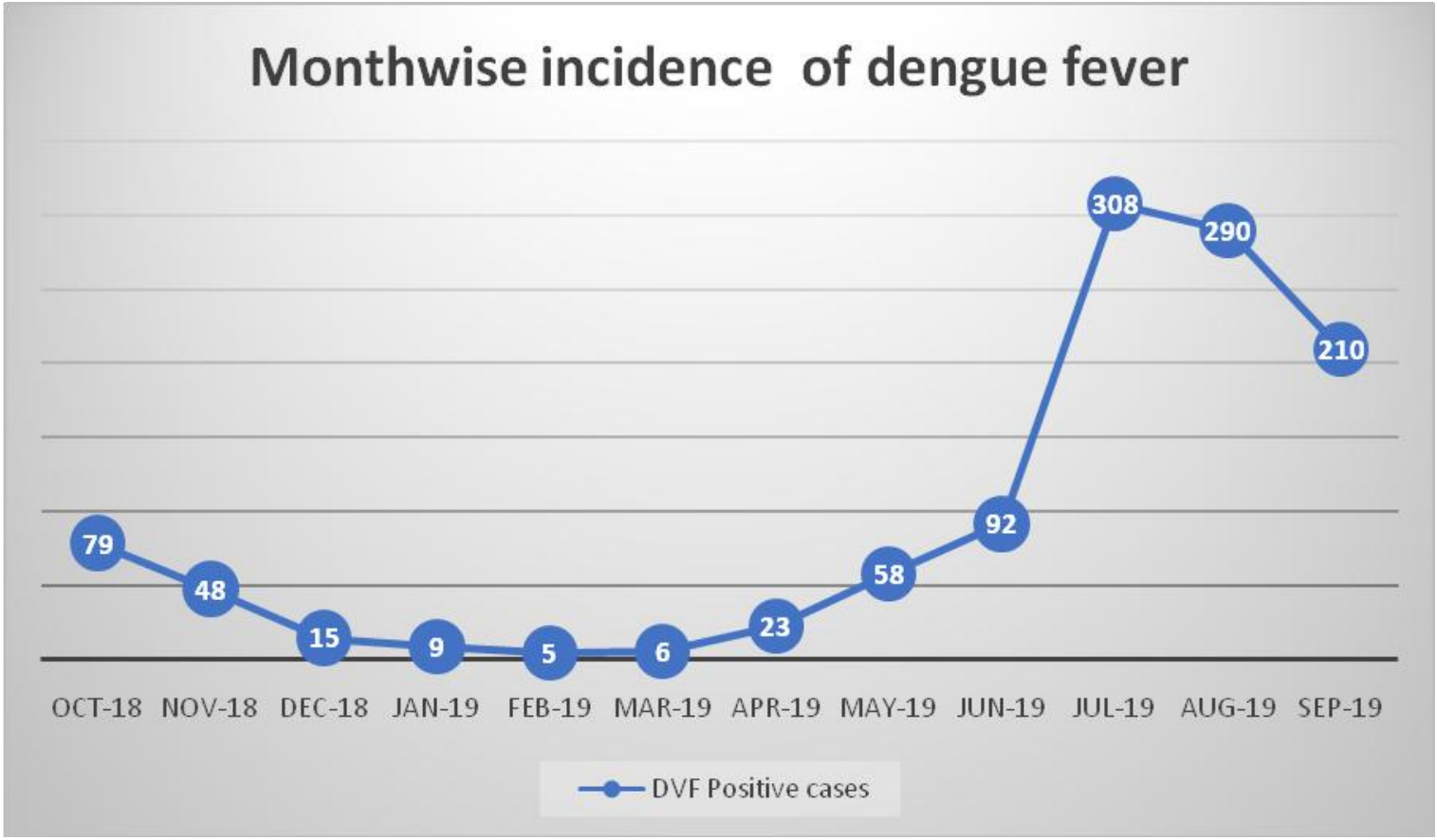

Patients belonging to age group of 0-30 years were $70.16 \%$ (210) and out of 299 patients, $63.21 \%$ were males ${ }^{7}$. Another study done in 2014 in Ernakulam too found similar distribution Dengue viral fever between male and female patients; out of 341 reported cases of dengue, $60.70 \%$ were males and $39.29 \%$ were females ${ }^{8}$.

Insofar as NS-1 laboratory assay is concerned; a study done in Delhi in 2010 recommended NS1 assay for early diagnosis of dengue infection $^{12}$. Also, in another study done in Chandigarh, it was concluded that NS1 antigen is an early diagnostic marker that is feasible in a routine diagnostic laboratory ${ }^{9}$.

Further a study done in Florida in 2017 recommended NS1 assays for early detection of dengue virus. In 2017 in a study done in Singapore, it was found that Viral detection diagnostic methods such as NS1 antigen assays are generally used within the first week of illness onset, whereas dengue serology testing is most appropriate after that time frame $^{10}$.

The National Guidelines for Clinical management of Dengue fever released by the Govt of India in 2014 characterizes the usefulness of Ns1 assays as follows: Dengue NS1 antigen, a highly conserved glycoprotein which is produced in both membraneassociated and secretion forms, is abundant in the serum of patients during the early stages of DENV infection.

It has been found to be useful as a tool for the diagnosis of acute dengue infections. It is a simple test that is more specific and shows high sensitivity. The present study too found the efficacy of NS1 assay as a tool for early detection of dengue viral infection when used within 5 days of fever and $\operatorname{IgM}$ antibody was more appropriate after 5 days. 


\section{References}

1. The Organization, Implementation, and Functioning of Dengue Surveillance in India—A Systematic Scoping ReviewEva Pilot, Vasileios Nittas, and Gudlavalleti Venkata S Murthy.

2. P.S. Santosh Kumar, M.C. Arjun, S.K. Gupta, B. Nongkynrih Malaria, dengue and chikungunya in India - an update Indian J Med Spec, 9 (1) (2018), pp. 25.

3. Chakravarti, R. Arora, C. Luxemburger Fifty years of dengue in India Trans R Soc Trop Med Hyg, 106 (2012), pp. 273282.

4. Victor TJ, Malathi M, Asokan R, Padmanaban P. Laboratory-based dengue fever surveillance in Tamil Nadu, India. Indian J Med Res 2007;126:112-

5. Chakravarti A, Kumaria R. Ecoepidemiological analysis of dengue infection during an outbreak of dengue fever, India. Virol J 2005; 2: 32.

6. Sci Total Environ. 2019 Jan 10;647:794805.

doi:

10.1016/j.scitotenv.2018.08.019. Epub 2018 Aug 3.

7. Lai YH. Biomed Eng Online. 2018 Nov 6;17(Suppl 2):148. doi: 10.1186/s12938018-0575-4.

8. Babita Bisht, Roop Kumari, BN Nagpal, Himmat Singh, Sanjeev Kumar Gupta, AK Bansal and NR Tulinternational Journal of Mosquito Research 2019; 6(2):
11-18.

9. Salam N Int J Med Res Health Sci 2018, 7(12): 149-155.

10. Salam, N Int J Med Res Health Sci 2019, 8(2): 8-12.

11. Mehta SR, Bafna TA, Pokale AB. Demographic and clinical spectrum of dengue patients admitted in a tertiary care hospital. Med J DY Patil Vidyapeeth 2018; 11: 128-31.

12. Antony J, Celine T M. A descriptive study on dengue fever reported in a Medical College Hospital. Sahel Med J 2014; 17: 83-6.

13. Datta, Simran and Wattal, Chand. (2010). Dengue NS1 antigen detection: A useful tool in early diagnosis of dengue virus infection. Indian journal of medical microbiology. 28. 107-10. 10.4103/02550857.62484 .

14. Singh M.P., Majumdar M., Singh G., Goyal K., Preet K., Sarwal A., Mishra B., Ratho R.K. (2010) Diagnostic Microbiology and Infectious Disease, 68(1), pp. 50-54.

15. Jason H. Ambrose, Shamala Devi Sekaran, and Azliyati Azizan Journal of Tropical Medicine Volume 2017, Article ID 8072491, 6 Pp.

16. Chan, Hui Bin Yvonne et al., "Definitive tests for dengue fever: when and which should I use?" Singapore medical journal vol. $58, \quad 11$ (2017): 632-635. doi:10.11622/smedj.2017100.

\section{How to cite this article:}

Ruksana Ahmed, Fatima Amatullah, Jyothi Allamsette and Suneetha Padala. 2019. Dengue Fever-A Seasonal Malady. Int.J.Curr.Microbiol.App.Sci. 8(11): 634-639. doi: https://doi.org/10.20546/ijcmas.2019.811.076 